Slavica

bruxellensia

\section{Slavica bruxellensia}

Revue polyphonique de littérature, culture et histoire

slaves

$11 \mid 2015$

Littérature et philosophie

\title{
Le Théâtre après 1989. ReflActions du théâtre en Europe médiane, sous la direction de Flock S.
}

\section{Cécile Bocianowski}

\section{OpenEdition}

\section{Journals}

Édition électronique

URL : http://journals.openedition.org/slavica/1695

DOI : 10.4000/slavica.1695

ISSN : 2034-6395

\section{Éditeur}

Université libre de Bruxelles - ULB

\section{Référence électronique}

Cécile Bocianowski, «Le Théâtre après 1989. ReflActions du théâtre en Europe médiane, sous la direction de Flock S. », Slavica bruxellensia [En ligne], 11 | 2015, mis en ligne le 25 février 2015, consulté le 22 septembre 2020. URL : http://journals.openedition.org/slavica/1695 ; DOI : https://doi.org/10.4000/ slavica. 1695

Ce document a été généré automatiquement le 22 septembre 2020.

\section{cc) $($ ) $\Theta$}

Les contenus de Slavica bruxellensia sont mis à disposition selon les termes de la Licence Creative Commons Attribution - Pas d'Utilisation Commerciale - Pas de Modification 3.0 France. 


\section{Le Théâtre après 1989. ReflActions du théâtre en Europe médiane, sous la direction de Flock S.}

Cécile Bocianowski

\section{RÉFÉRENCE}

Le Théâtre après 1989. ReflActions du théâtre en Europe médiane, sous la direction de Flock S., Prague, Institut des Arts et du Théâtre, 2012, 146 p. 
1 L'ouvrage, dirigé par Sarah Flock, jeune docteur de l'ULB et de l'Université Charles de Prague, est le fruit de la conférence organisée par le Centre d'Études Tchèques de l'ULB en novembre 2009, à l'occasion des vingt ans de la chute du Mur de Berlin, sur le théâtre des pays " médians " après le tournant politique et social de 1989. Le sous-titre, «RéflActions du théâtre en Europe médiane " suggère le questionnement qui a donné naissance à cette réflexion polyphonique, celui de la place et de la fonction d'un théâtre qui est passé de la contestation et de la censure à la liberté, la démocratie et le libéralisme, et ce, dans les pays tchèque, slovaque, polonais, slovène, roumain, hongrois et bulgare.

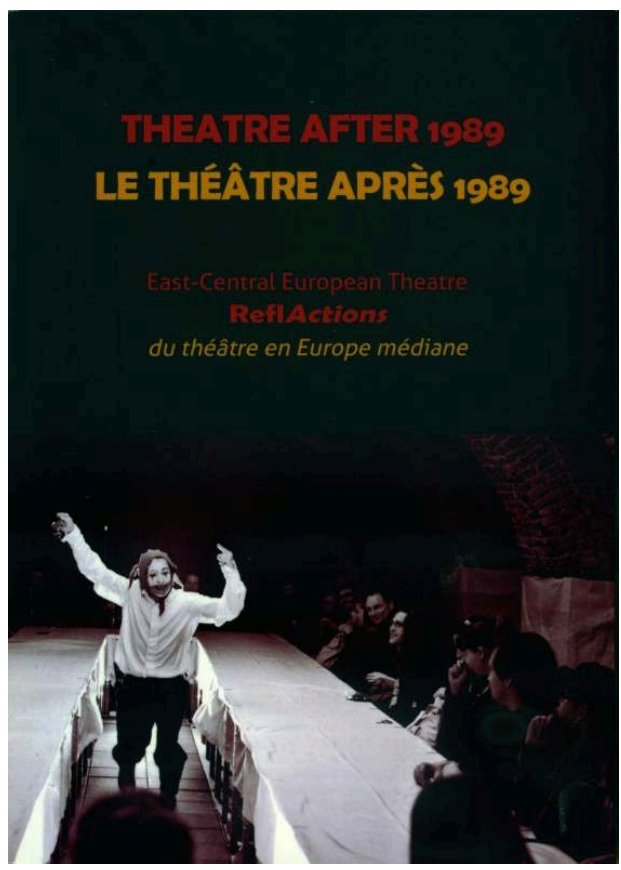

2 Si la réflexion sur les effets du changement de régime dans la littérature d'Europe centrale, orientale et sud-orientale a donné lieu à plusieurs ouvrages récents consacrés à l'après 1989 dans le domaine universitaire francophone, ceux-ci étaient principalement consacrés à la prose. À ce titre, l'ouvrage issu du colloque de Bruxelles constitue une publication pertinente. Par ailleurs, il s'inscrit dans la lignée d'ouvrages anglophones abordant le théâtre de cet espace-temps qu'il vient enrichir en se focalisant sur les interactions entre scène et révolution.

3 Rédigées pour la plupart en anglais, pour certaines en français suivies d'un résumé dans l'autre langue, les diverses contributions, chacune consacrée à un pays particulier, laissent place dans le dernier article à une perspective comparatiste qui s'interroge sur la mondialisation du théâtre.

4 L'ouvrage, composé de dix articles reprenant les communications des orateurs de la première journée de colloque, s'ouvre sur une attention particulière portée au théâtre tchèque, auquel sont consacrés les quatre premiers articles. Le premier met en avant le rôle engagé des théâtres lors de la révolution de velours, et le parcours de Petr Lébl, metteur en scène évoluant principalement dans les cercles alternatifs avant la révolution pour ensuite travailler avec le plus prestigieux théâtre tchèque. La deuxième contribution présente les questionnements et inquiétudes suscitées par le changement de 1989 quant au statut esthétique du théâtre qui pourrait tourner au divertissement, à son statut économique et à son rapport au politique. Le troisième article livre une réflexion sur les 20 ans des changements politiques, réflexion nourrie par le témoignage de l'auteure ayant participé à l'action « Théâtre et révolution » en 1989, par les discussions qui ont eu lieu lors de colloques en 2009 à Prague ainsi que par les réflexions sur la trilogie du metteur en scène Vladimir Morávek, s'attaquant à l'héritage du nazisme et du communisme. La dernière communication consacrée au domaine tchèque nous livre quant à elle un point de vue sur la production actuelle du théâtre tchèque, en nous présentant l'esthétique répandue du théâtre hybride qui, 
malgré le faible soutien de la part de la politique culturelle tchèque, connaît le succès à l'étranger.

5 Ce premier temps consacré à la création tchèque est suivie des articles au sujet d'autres domaines géographiques, à commencer par la Slovénie, représentée par un professeur d'histoire du théâtre qui partage avec le lecteur, par le biais de sa pratique pédagogique et didactique, ses expériences face à la génération d'étudiants post-1989, victimes de la révision de l'histoire telle qu'elle a été enseignée sous le régime communiste; il présente ainsi les méthodes d'enseignement qu'il a développées en conséquence. Les deux articles consacrés à la création roumaine abordent la question du théatre après 1989 d'une perspective plus esthétique, le premier en posant la question du modèle dominant de mise en scène en Roumanie, nostalgique d'un âge d'or du théâtre modelé sur le celui des années 1920 et 1930, tandis que le second, consacré à la création roumaine de langue hongroise, s'attache à la relation entre corps, espace et image, soulignant la part grandissante de cette dernière dans le théâtre contemporain. S'ensuivent les articles traitant des domaines bulgares et enfin polonais, tous deux écrits par des dramaturges de formation, qui présentent, l'une un panorama de la création bulgare après 1989 qui cède à la tentation du théâtre commercial, l'autre un questionnement du sens du politique dans les arts en Pologne après la chute du Mur de Berlin, et la place du corps par rapport au politique. La dernière communication, enfin, propose une synthèse par la lecture des dramaturgies post-1989 à travers une grille d'analyse établie par la critique et dégageant les motifs communs aux pays concernés.

6 C'est donc un tableau éclectique tant par ses approches, ses problématiques que ses perspectives choisies que nous livre Le Théâtre après 1989. Le lecteur peut ainsi, au gré de ses affinités, puiser dans ces témoignages, analyses et synthèses pour enrichir ses connaissances du théâtre d'Europe médiane, découvrant les acteurs, les productions et les lieux de la révolution théâtrale qui a durablement modifié le paysage théâtral de ces pays.

7 On pourra reprocher à l'ouvrage de ne pas avoir reproduit toutes les communications qui ont constitué le colloque et que l'introduction du recueil ne fait que mentionner, sans les partager avec le lecteur, ne lui laissant entre les mains qu'une sélection de ce qui a constitué le dialogue croisé du colloque. D'autant plus que ceux-ci proposaient une perspective comparatiste et transversale qui aurait pu compléter les perspectives adoptées dans la sélection et qui n'est que brièvement abordée dans la dernière communication.

\section{AUTEURS}

\section{CÉCILE BOCIANOWSKI}

Doctorante en Littérature comparée à l'Université Paris IV-Sorbonne (France) ; assistante à l'Université Libre de Bruxelles (Belgique) ; membre du comité de rédaction de Slavica Bruxellensia 